\title{
The role of brain perivascular space burden in early-stage Parkinson's disease
}

Ting Shen ${ }^{1,2,3}$, Yumei Yue ${ }^{4}$, Shuai Zhao ${ }^{2}$, Juanjuan Xie ${ }^{1,2}$, Yanxing Chen ${ }^{2}$, Jun Tian ${ }^{2}$, Wen Lv ${ }^{4}$, Chun-Yi Zac Lo ${ }^{5}$, Yi-Cheng Hsu ${ }^{6}$, Tobias Kober $\mathbb{D}^{7}$, Baorong Zhang ${ }^{2 凶}$ and Hsin-Yi Lai $\mathbb{D}^{1,3,4 凶}$

Perivascular space (PVS) is associated with neurodegenerative diseases, while its effect on Parkinson's disease (PD) remains unclear. We aimed to investigate the clinical and neuroimaging significance of PVS in basal ganglia (BG) and midbrain in earlystage PD. We recruited 40 early-stage PD patients and 41 healthy controls (HCs). Both PVS number and volume were calculated to evaluate PVS burden on $7 \mathrm{~T}$ magnetic resonance imaging images. We compared PVS burden between PD and $\mathrm{HC}$, and conducted partial correlation analysis between PVS burden and clinical and imaging features. PD patients had a significantly more serious PVS burden in BG and midbrain, and the PVS number in BG was significantly correlated to the PD disease severity and L-dopa equivalent dosage. The fractional anisotropy and mean diffusivity values of certain subcortical nuclei and white matter fibers within or nearby the BG and midbrain were significantly correlated with the ipsilateral PVS burden indexes. Regarding to the midbrain, the difference between bilateral PVS burden was, respectively, correlated to the difference between fiber counts of white fiber tract passing through bilateral substantia nigra in PD. Our study suggests that PVS burden indexes in BG are candidate biomarkers to evaluate PD motor symptom severity and aid in predicting medication dosage. And our findings also highlight the potential correlations between PVS burden and both grey and white matter microstructures.

npj Parkinson's Disease (2021)7:12 ; https://doi.org/10.1038/s41531-021-00155-0

\section{INTRODUCTION}

Perivascular spaces (PVSs) are fluid-filled spaces surrounding blood vessels that course from the brain surface through brain parenchyma ${ }^{1}$. It's a normal anatomical structure in the central nervous system, commonly observed in basal ganglia (BG), white matter centrum semiovale, midbrain and hippocampus ${ }^{2}$. Although the results of PVS studies remained controversial, the PVS burden had been shown to correlate with aging, cognitive capacity ${ }^{3,4}$, sleep quality ${ }^{5}$, and depressive states ${ }^{6}$. It was described as a characteristic of neurological diseases including, small vessel disease ${ }^{7}$, Alzheimer's disease $(A D)^{8}$, multiple sclerosis ${ }^{9}$, as well as Parkinson's disease (PD) ${ }^{10,11}$.

$\mathrm{PD}$ is a chronic neurodegenerative disorder, characterized by motor symptoms (resting tremor, bradykinesia, rigidity, and gait abnormalities) and non-motor symptoms (depression, anxiety, cognition decline, etc. $)^{12}$. The pathological hallmarks of PD are dopaminergic neuron degeneration and abnormal a-synuclein deposition in the substantia nigra $(\mathrm{SN})^{13}$. Recent studies indicated that the PVS system played a disease-modifying role in $\mathrm{PD}^{10,11,14,15}$. Since the PVS system is involved in the glymphatic drainage system to eliminate metabolic waste ${ }^{16,17}$, and there was evidence showing that PVS system helped drive out soluble proteins involved in neurodegenerative diseases from the brain interstitial fluid ${ }^{18}$, we also assume that PVS participates in the clearance of abnormal a-synuclein ${ }^{19}$ and its dysfunction may aggravate the pathology of PD.
Enlarged PVS became visible and measurable through magnetic resonance imaging (MRI) techniques. PVS burden was defined as indexes of PVS quantification to represent severity of PVS enlargement, including PVS number and volume ${ }^{20}$. Previous studies reported that PVS might contribute to the characteristic asymmetry of motor symptoms ${ }^{10}$, and could be a useful neuroimaging biomarker for evaluating cognitive decline in $\mathrm{PD}^{10,15}$. The physiological and pathophysiological mechanisms of PVS may be reflected in its neighboring white matter, as PVS burden had shown correlation with white matter hyperintensity ${ }^{21}$.

These studies partly explained the significance of PVS, however, it was previously subject to the limitation of MRI resolution. Only PVSs with larger diameters were visible, and most studies focused primarily on the potential pathogenicity of enlarged PVSs. Due to the higher resolution and signal-to-noise ratio (SNR) of 7 Tesla (7T) MRI, more small size PVSs can be detected and the PVS numbers were markedly higher than conventional 1.5 or $3 \mathrm{~T}$ MRI studies ${ }^{1}$. Therefore, the small PVS should also be taken into account in studies of neurodegenerative diseases, which could alter the correlations between PVS burden and clinical features compared to previous studies ${ }^{1}$.

This study aimed to compare the PVS burden in early-stage PD to those in healthy controls ( $\mathrm{HCs}$ ), with the influence of smaller PVSs included. We performed a quantitative evaluation of PVS burden on 7T MRI images to explore the prevalence of PVS and characterize the correlation patterns of PVS burden with clinical

\footnotetext{
'Department of Neurology of the Second Affiliated Hospital, Interdisciplinary Institute of Neuroscience and Technology, Key Laboratory of Medical Neurobiology of Zhejiang Province, Zhejiang University School of Medicine, Zhejiang University, Hangzhou, China. ${ }^{2}$ Department of Neurology of the Second Affiliated Hospital, Zhejiang University School of Medicine, Zhejiang University, Hangzhou, China. ${ }^{3}$ College of Biomedical Engineering and Instrument Science, Key Laboratory for Biomedical Engineering of Ministry of Education, Zhejiang University, Hangzhou, China. ${ }^{4}$ Department of Neurology of Sir Run Run Shaw Hospital, Zhejiang University School of Medicine, Zhejiang University, Hangzhou, China. ${ }^{5}$ Institute of Science and Technology for Brain-Inspired Intelligence, Fudan University, Shanghai, China. ${ }^{6}$ MR collaboration NE Asia, Siemens Healthcare, Shanghai, China. ${ }^{7}$ Advanced Clinical Imaging Technology, Siemens Healthcare, Lausanne, Switzerland. ${ }^{凶}$ email: brzhang@zju.edu.cn; laihy@zju.edu.cn
} 
and imaging features to further understand its pathophysiological mechanism in PD.

\section{RESULTS}

\section{Participant characteristics}

The demographic and clinical characteristics of the PD and HC participants are shown in Table 1. There were no significant differences between the PD and HC groups in age, sex, education, Hamilton Rating Scale for Anxiety (HAMA) score, Mini-Mental State Examination (MMSE) score, Montreal Cognitive Assessment (MoCA) score, Parkinson Neuropsychometric Dementia Assessment (PANDA) score, or prevalence of vascular risk factors such as hypertension, hyperlipidemia, diabetes mellitus, and smoking. The PD patients had significantly higher Hamilton Rating Scale for Depression (HAMD) score than the HC participants $(p=0.009)$, indicating the depressive state was more severe in the PD patients.

\section{Distribution of PVSs}

Although our $3 \mathrm{~T}$ MRI sequence already achieved a relatively high resolution, 7T MRI images could expose PVSs more clearly, particularly for small size PVSs due to its much higher SNR compared to $3 \mathrm{~T} \mathrm{MRI} \mathrm{images} \mathrm{(Fig.} \mathrm{1a).} \mathrm{The} \mathrm{PVS} \mathrm{quantification} \mathrm{was}$ performed by a single reader (T.S.) blind to the participants' clinical status. Before the formal study, reader (T.S.) was trained by a senior researcher (Y.Y.) on a training set of 20 cases $^{21}$. Intra-class

Table 1. Demographic and clinical characteristics of participants.

\begin{tabular}{llll}
\hline & PD $(n=40)$ & HC $(n=41)$ & $p$ \\
\hline Age (years) & $52.5 \pm 1.2$ & $49.8 \pm 2.0$ & 0.242 \\
Sex, male (\%) & 47.5 & 51.2 & 0.738 \\
Education (years) & $8.8 \pm 0.7$ & $10.8 \pm 0.7$ & 0.059 \\
Hypertension (\%) & 25.0 & 19.5 & 0.553 \\
Diabetes mellitus (\%) & 5.0 & 4.9 & 0.626 \\
Hyperlipidemia (\%) & 0.0 & 4.9 & 0.485 \\
Smoking (\%) & 17.5 & 19.5 & 0.816 \\
Disease duration (years) & $5.4 \pm 1.0$ & - & - \\
MDS-UPDRS I & $5.5 \pm 0.5$ & - & - \\
MDS-UPDRS II & $8.4 \pm 0.6$ & - & - \\
MDS-UPDRS III & $23.4 \pm 2.0$ & - & - \\
MDS-UPDRS IV & $1.9 \pm 0.5$ & - & - \\
MDS-UPDRS total & $39.1 \pm 3.0$ & - & - \\
H-Y stage & $1.8 \pm 0.1$ & - & - \\
LEDD (mg) & $535.0 \pm 39.5$ & - & - \\
HAMA & $3.7 \pm 0.5$ & $2.7 \pm 0.5$ & 0.083 \\
HAMD & $5.8 \pm 0.6$ & $3.8 \pm 0.6$ & $0.009 * *$ \\
MMSE & $26.5 \pm 0.5$ & $27.3 \pm 0.4$ & 0.272 \\
MoCA & $22.6 \pm 0.8$ & $23.8 \pm 0.7$ & 0.285 \\
PANDA & $20.7 \pm 0.9$ & $21.3 \pm 1.1$ & 0.672 \\
\hline Resuls are expsse & 2. & & \\
\hline
\end{tabular}

Results are expressed as means \pm standard error of the mean for the continuous variables and as frequencies for the categorical variables. PD Parkinson's disease, $H C$ healthy control, MDS-UPDRS Movement Disorder Society-sponsored revision of the Unified Parkinson's Disease Rating Scale, $\mathrm{H}-\mathrm{Y}$ stage Hoehn \& Yahr stage, LEDD L-dopa equivalent daily dose, HAMA Hamilton Rating Scale for Anxiety, HAMD Hamilton Rating Scale for Depression, MMSE Mini-Mental State Examination, MoCA Montreal Cognitive Assessment, PANDA Parkinson neuropsychometric dementia assessment.

**Indicates $p<0.01$ correlation coefficients between two readers reached 0.81 for PVSs in BG and 0.89 for PVSs in midbrain.

In this study, the PVS burden indexes, PVS number and PVS volume, in the right-hemispheric $B G\left(B G_{R}\right)$, left-hemispheric $B G$ $\left(B G_{L}\right)$, right-hemispheric midbrain $\left(\mathrm{Mid}_{R}\right)$, and left-hemispheric midbrain $\left(\right.$ Mid $\left._{L}\right)$ were shown in Table 2 . Both PVS number and volume in $B G_{R}$ were significantly larger in the $P D$ group than those in the HC group $(p=0.035$ for number, $p=0.015$ for volume). And the PVS number in bilateral midbrain $\left(p=0.042\right.$ for $\operatorname{Mid}_{R}, p=0.018$ for $\left.\operatorname{Mid}_{L}\right)$ and the PVS volume in $\operatorname{Mid}_{R}(p=0.041)$ were significantly larger in the PD group compared to the HC group, as shown in Fig. 2.

\section{Correlation analysis with clinical features}

After regressing out the effect of age, sex, and total brain volume, the PVS number of $B G_{L}$ was positively correlated with Movement Disorder Society-sponsored revision of the Unified Parkinson's Disease Rating Scale (MDS-UPDRS) II subscore (partial correlation coefficients $(p c c)=0.481, p=0.003)$, III subscore $(p c c=0.369$, $p=0.025)$, IV subscore $(\mathrm{pcc}=0.402, p=0.014)$, and total score $(\mathrm{pcc}=0.451, p=0.005)$ in the PD group (Fig. 3a-d), while the PVS volume of $B G_{L}$ was not correlated to MDS-UPDRS scores. The PVS number of $B G_{R}$ was positively correlated to $L$-dopa equivalent daily dose (LEDD) (pcc $=0.347, p=0.036$, Fig. 3e). However, the PVS burden indexes were not correlated to HAMA, HAMD, MMSE, MoCA, or PANDA scores.

In the HC group, the PVS number of $B G_{L} \quad(p c c=0.475$, $p=0.007)$, the PVS number of $\operatorname{Mid}_{\mathrm{L}}(\mathrm{pcc}=0.511, p=0.003)$ and the PVS volume of $\mathrm{Mid}_{\mathrm{L}}$ ( $\mathrm{pcc}=0.429, p=0.016$ ) were positively correlated with the HAMD score after regressing out the effect of age, sex, and total brain volume (Fig. $3 f-h)$. Furthermore, the PVS burden indexes in $\mathrm{Mid}_{\mathrm{L}}$ were negatively correlated to the PANDA score (pcc $=-0.398, p=0.030$ for PVS number; $p c c=-0.471$, $p=0.009$ for PVS volume), as shown in Fig. $3 \mathrm{i}$, j.

\section{Correlation analysis with FA and MD values}

Results of the regions of interest (ROI)-based analysis showed that the PD group had significantly lower fractional anisotropy (FA) value in right-hemispheric ventral caudate $(\mathrm{vCa})(p=0.031)$ and higher FA value in left-hemispheric globus pallidus (GP) ( $p=$ 0.031 ), as well as higher mean diffusivity (MD) values in bilateral vCa $(p=0.0003$ for right-hemisphere, $p=0.030$ for left-hemisphere), bilateral dorsal caudate (dCa) $(p=0.0006$ for righthemisphere, $p=0.021$ for left-hemisphere), right-hemispheric ventromedial putamen (vmPu) $(p=0.027)$, and right-hemispheric $\mathrm{GP}(p=0.049)$. In the fiber of interest (FOI)-based analysis, we also found significantly higher FA value in right-hemispheric anterior limb of internal capsule (ALIC) $(p=0.005)$, as well as higher MD value in right-hemispheric ALIC $(p=0.005)$ and lower MD values in left-hemispheric cerebral peduncle (CP) $(p=0.029)$ in PD group compared to HC group (Fig. $4 a, b)$. The results of right vCa and right $\mathrm{dCa}$ were survived after false discovery rate (FDR) correction.

Correlations between the FA values and the PVS burden indexes were presented in Fig. 4c. The FA value of subcortical nuclei, such as the right-hemispheric $\mathrm{SN}$ within midbrain was negatively correlated with the PVS number for the $\operatorname{Mid}_{R}(p c c=-0.342$, $p=0.044)$ in PD group, whereas a similar presentation was also found in $\mathrm{HC}$ group ( $\mathrm{pcc}=-0.322, p=0.049$ ). Regarding to the white matter fibers, the FA value of the left-hemispheric $C P$ was negatively correlated with the PVS volume of $\mathrm{Mid}_{\mathrm{L}}(\mathrm{pcc}=-0.341$, $p=0.045$ ) in the PD group. And in the HC group, the PVS number of $B G_{R}$ was positively correlated with $F A$ values of righthemispheric external capsule $(E C)$ ( $p c c=0.369, p=0.023$ ).

Correlations between MD values and PVS burden indexes were presented in Fig. 4d. The MD value of left-hemispheric SN was positively correlated with the PVS volume of $\operatorname{Mid}_{\mathrm{L}}(\mathrm{pcc}=0.359$, $p=0.034$ ) in the PD group. In the HC group, the MD value of 
a

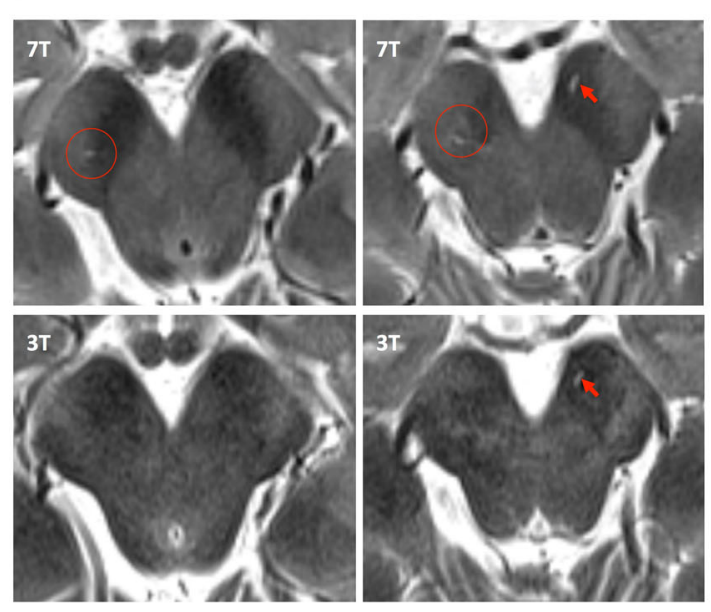

b

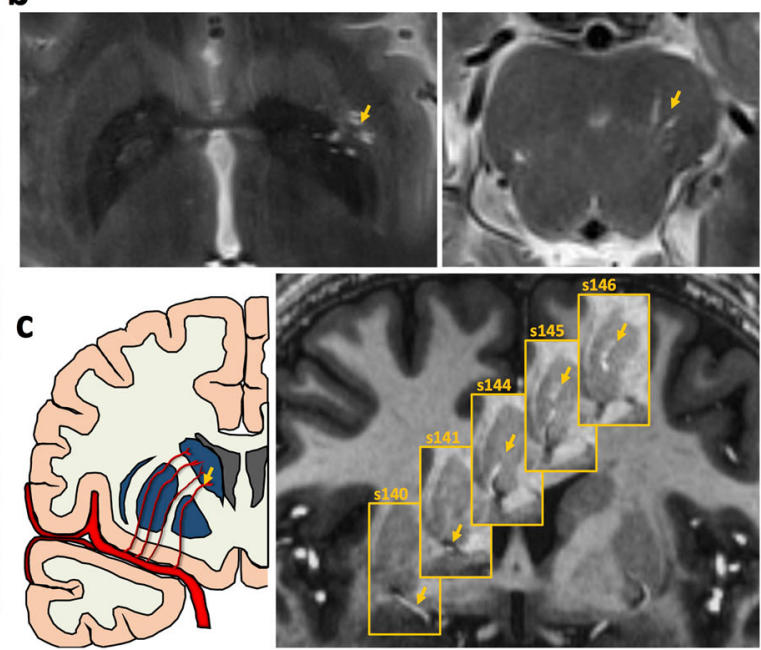

Fig. 1 Visualization of the perivascular space. a Comparison of detectable PVS in one subject on $7 \mathrm{~T}$ images and $3 \mathrm{~T}$ images. $\mathbf{b}$ PVSs in the BG and midbrain on T2 images. c Visualization of the PVS system and its spatially corresponding lenticulostriate artery in the BG on MP2RAGE imaging.

\begin{tabular}{|c|c|c|c|}
\hline & $\mathrm{PD}(n=40)$ & $\mathrm{HC}(n=41)$ & $p$ \\
\hline \multicolumn{4}{|l|}{$B G_{R}$} \\
\hline Number & $12.4 \pm 0.8$ & $9.81 \pm 0.7$ & $0.035^{*}$ \\
\hline Volume & $41.5 \pm 3.9$ & $29.1 \pm 2.7$ & $0.015^{*}$ \\
\hline \multicolumn{4}{|l|}{$B G_{L}$} \\
\hline Number & $11.9 \pm 0.8$ & $11.1 \pm 0.8$ & 0.555 \\
\hline Volume & $36.9 \pm 3.3$ & $35.6 \pm 3.4$ & 0.921 \\
\hline \multicolumn{4}{|l|}{$\operatorname{Mid}_{R}$} \\
\hline Number & $8.2 \pm 0.6$ & $6.2 \pm 0.6$ & $0.042 *$ \\
\hline Volume & $13.5 \pm 1.2$ & $9.6 \pm 1.4$ & $0.041^{*}$ \\
\hline \multicolumn{4}{|l|}{$\operatorname{Mid}_{L}$} \\
\hline Number & $6.6 \pm 0.5$ & $4.6 \pm 0.5$ & $0.018^{*}$ \\
\hline Volume & $9.1 \pm 1.0$ & $6.8 \pm 1.1$ & 0.145 \\
\hline
\end{tabular}

right-hemispheric GP was positively correlated with the PVS volume of $\mathrm{BG}_{\mathrm{R}}(\mathrm{pcc}=0.346, p=0.033)$. In regard to the white matter fibers, the PVS volume of $B G_{L}$ was positively correlated with the MD value of left-hemispheric retrolenticular part of internal capsule (RPIC) ( $\mathrm{pcc}=0.393, p=0.019)$ in the PD group. In the $\mathrm{HC}$ group, the MD value of right-hemispheric ALIC was positively correlated with the PVS number $(p c c=0.342, p=0.035)$ and volume ( $\mathrm{pcc}=0.330, p=0.043$ ) of $\mathrm{BG}_{\mathrm{R}}$. The MD value of righthemispheric EC was positively correlated to the PVS number of $B G_{R}(p c c=0.330, p=0.043)$, and the MD value of left-hemispheric EC was positively correlated to the PVS number $(\mathrm{pcc}=0.363, p=$ $0.025)$ and volume $(p c c=0.337, p=0.039)$ of $B G_{L}$.

\section{Correlation analysis with white matter fibers}

There was no significant difference of the fiber counts of white fiber tract passing through the nuclei within BG and midbrain between PD and HC groups, and we did not find significant correlations between PVS burden and fiber counts. Regarding to
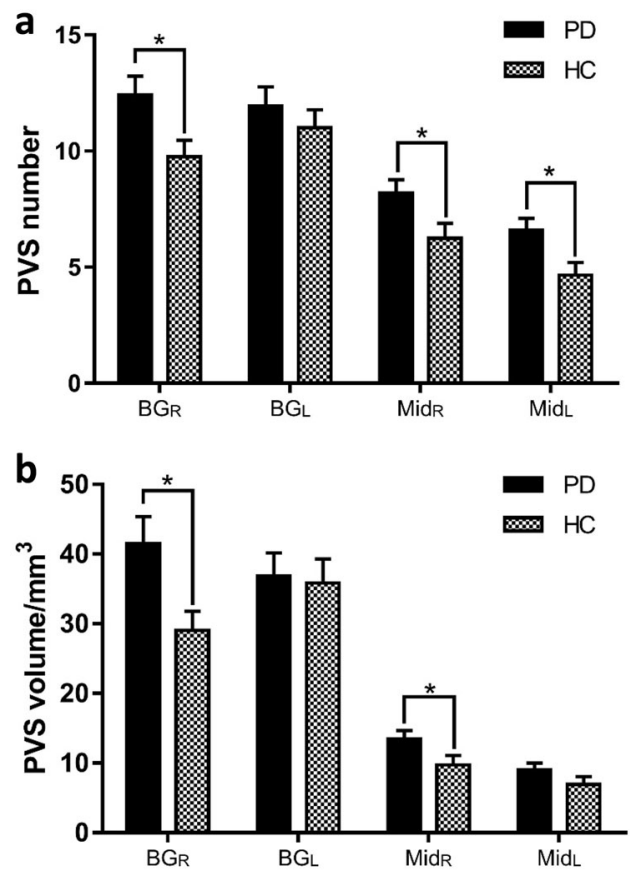

Fig. 2 Comparison of the perivascular space burden between PD and HC groups. a PVS number and b PVS volume in BG and midbrain (Mid). *Indicates $p<0.05$. The results are presented as means \pm standard error of mean.

the BG region, the difference between bilateral PVS burden indexes (both PVS number and volume) were not significantly correlated to the difference between fiber counts of white matter fiber tract passing through bilateral subcortical nuclei, including the vCa, dCa, vmPu, dorsolateral putamen (dIPu), GP, and whole $B G$ in both PD and HC groups (only results of whole BG in PD was shown in Fig. 5a). And with respect to the midbrain region, the difference between bilateral PVS burden indexes were, respectively, correlated to the difference between fiber counts of white fiber tract passing through bilateral SN $(\mathrm{pcc}=-0.344, p=0.043$ for PVS number; $\mathrm{pcc}=-0.351, p=0.038$ for PVS volume) after controlling for total brain volume, age, and sex in the PD group 

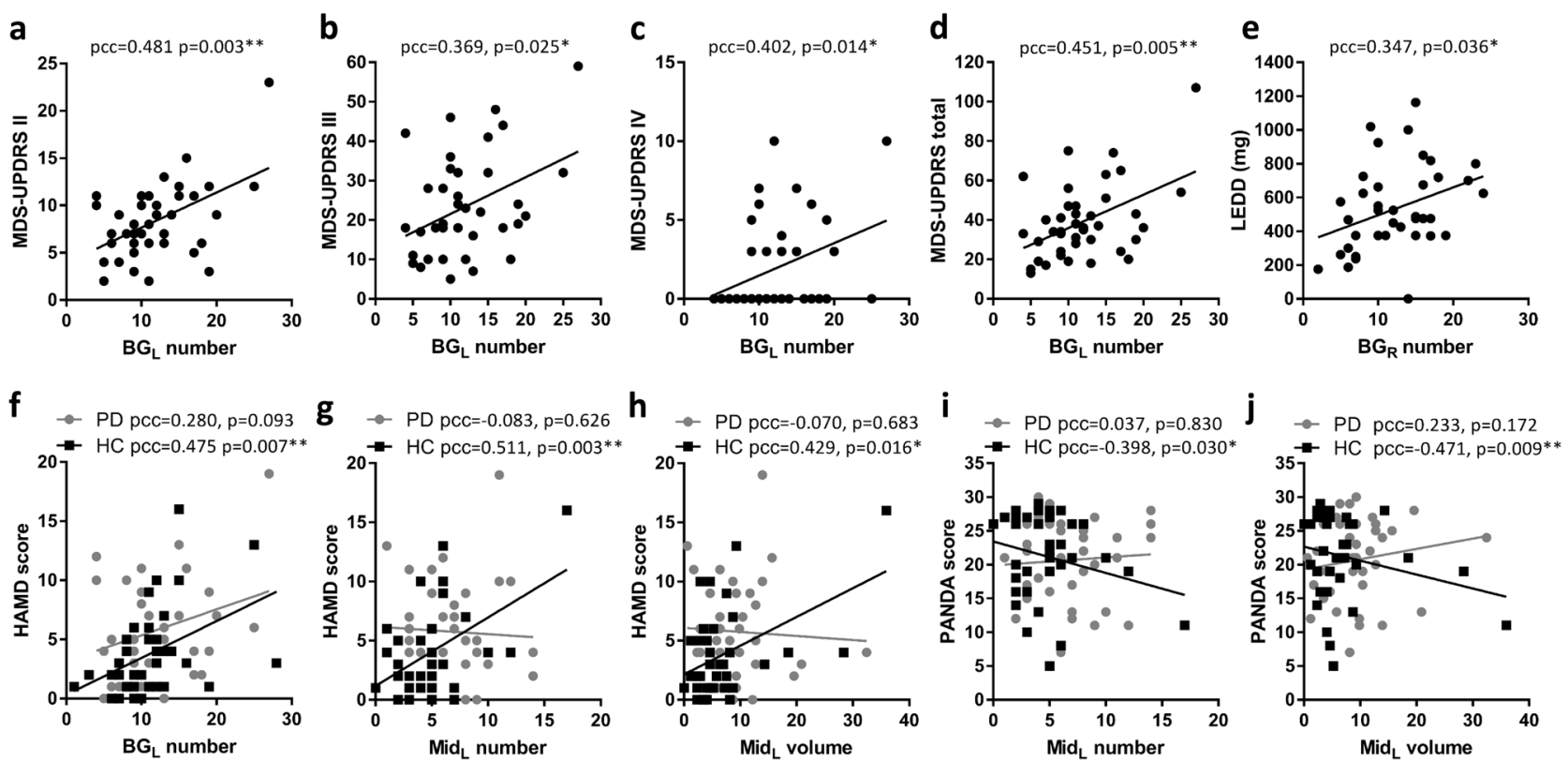

Fig. 3 Correlations of perivascular space burden indexes in BG and midbrain with clinical features. Correlations with a-d MDS-UPDRS scores and $\mathbf{e}$ LEDD in PD. Correlations with $\mathbf{f}-\mathbf{h}$ HAMD score in PD and HC. Correlation with $\mathbf{i}-\mathbf{j}$ PANDA score in PD and HC. *Indicates the partial correlation coefficient $(\mathrm{pcc})<0.05$ and ${ }^{* *}$ indicates pcc $<0.01$.

(Fig. 5b). Although there was no statistical significance, the $\mathrm{HC}$ group still showed a negative correlation trend.

\section{DISCUSSION}

In this study, we explored the PVS burden considering both number and volume in BG and midbrain in the early-stage PD patients. We detected significantly heavier PVS burden in PD group than the HC group on 7T MRI images, as well as provided fresh data to support the relationship between PVS burden and motor symptom severity in PD. Along with previous studies, we also found that PVS burden was significantly correlated with cognitive function and emotional status. Furthermore, changes of diffusion tensor imaging (DTI)-derived features of certain subcortical nuclei and white matter fibers were also associated with PVS burden.

Considering the potential functions of the PVS system, studies had proved that the PVS waste drainage system contributed to a larger portion of interstitial solutes clearance, such as amyloid beta $(A \beta)$, preventing $A \beta$ deposition that was thought to be pathogenic in $A D^{18,22}$. Similar to $A D$, abnormal aggregated protein $a$-synuclein that forms Lewy bodies or Lewy neuritis is the main neuropathological characteristics of $\mathrm{PD}^{13}$. PVS may also contribute to clearance of abnormal a-synuclein in the brain ${ }^{19}$. On the one hand, the failure of perivascular clearance might cause increased a-synuclein deposition, formation of Lewy pathology and damage of dopaminergic neurons, which might lead to modification of PD pathology and clinical features. On the other hand, dopaminergic neuronal degeneration might produce excessive metabolic wastes and accumulated in PVS, lead to perivascular blockage and heavier PVS burden, which might contribute to the pathological process in $\mathrm{PD}^{23}$. Unfortunately, previous studies linking PVS and dopamine transporter (DAT) uptake showed inconsistent results. Several case reports and case-control studies had shown that the existence of PVS might relate to lower DAT uptake ${ }^{24-26}$. And it was also proposed that enlarged PVSs would cause downstream dysfunction from the presynaptic nigrostriatal dopaminergic system and led to incomplete response to levodopa ${ }^{25,26}$. Other study showed no significant correlation between PVS burden and DAT positron emission tomography (PET) abnormality ${ }^{11}$.
To explain this phenomena, studies had identified two types of PVS according to the histological appearance of the surrounding tissue: type 1 with normal surrounding nervous tissue, and type 2 with surrounding rarefication and abnormal gliosis ${ }^{11,27}$, suggesting that these inconsistent results might be due to different types of detected PVS in these studies. The most commonly detected PVSs on MRI images have diameters $<3 \mathrm{~mm}$, are used to be considered as type 1 with normal physiological functions. Accordingly, the present study mainly focused on the potential role of these PVSs.

Previous studies mainly focused on reporting PD cases with enlarged PVS ${ }^{14,25,28,29}$ and studying the populations of PD patients without comparing to the normal controls ${ }^{15,30,31}$. We compared the PVS burden of PD group to those of HCs, and found a more severe PVS burden in PD. Another difference with previous studies was that those studies mainly focused on middle-stage and latestage PD patients with average of ages over 60 years old ${ }^{10,11}$, whereas we intended to investigate the PVS burden in early-stage PD patients with average of age about 50 years old. We observed more severe PVS burden in the PD group, therefore we revealed that the exacerbation of PVS burden was already existed in the early phase of PD disease process. Statistically significant differences were mainly found in PVS burden in the righthemisphere, the left hemisphere only showed an increasing trend in PD. To a certain extent, our results might provide new evidence for hemispheric lateralization among PD. The progress of PVS burden in PD might start from right-hemisphere, and irrelevant to symptom onset-side. Previous studies have pointed out the lateralization of certain brain function, the right-hemisphere is dominant for controlling limb position and posture, whereas the left-hemisphere is the dominant for appendicular movements ${ }^{32,33}$. The neurodegenerative process of PD also initials asymmetrically, might exhibit left-hemispheric predominance of subclinical nigrostriatal deficit ${ }^{34,35}$. Moreover, PD patients with freezing of gait were presented with more affected right-hemisphere circuitry, especially in the pedunculopontine nucleus within midbrain $^{36,37}$. However, the mechanism of lateralization for motor features and neuro-pathophysiology of PD still requires more evidence. In the present study, significant correlations between the PVS burden in left BG and MDS-UPDRS scores were also found 
a

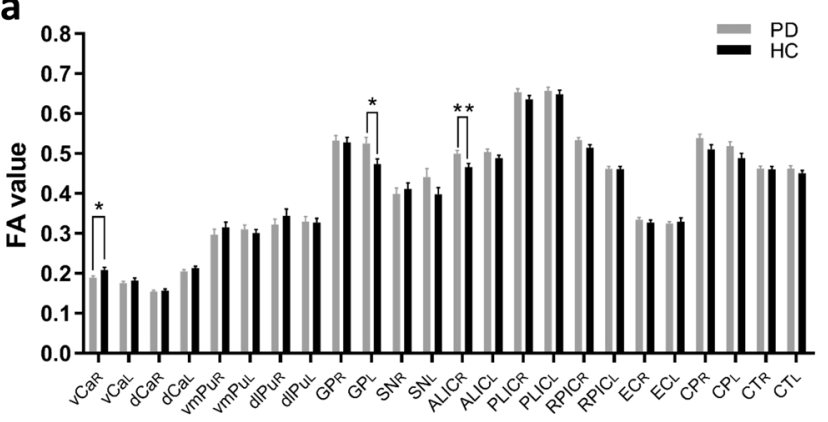

b

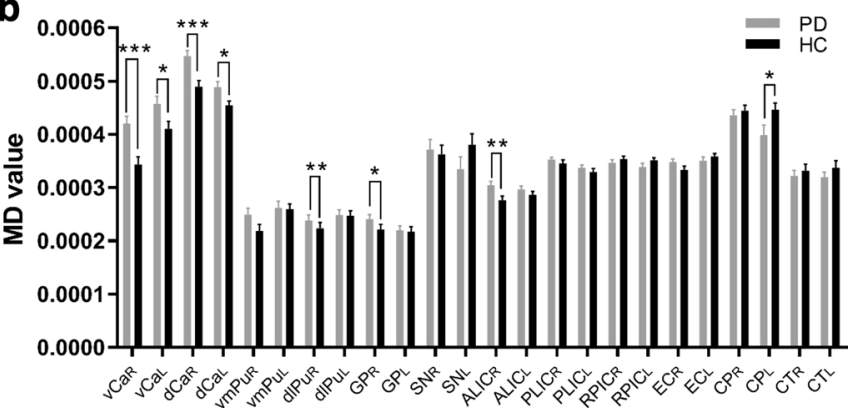

C

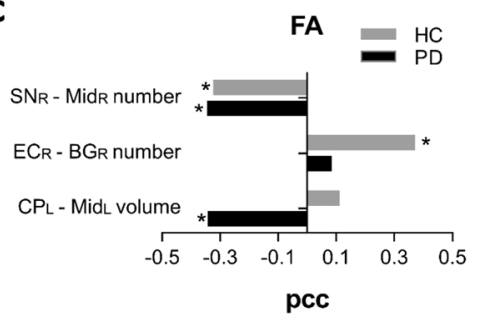

d

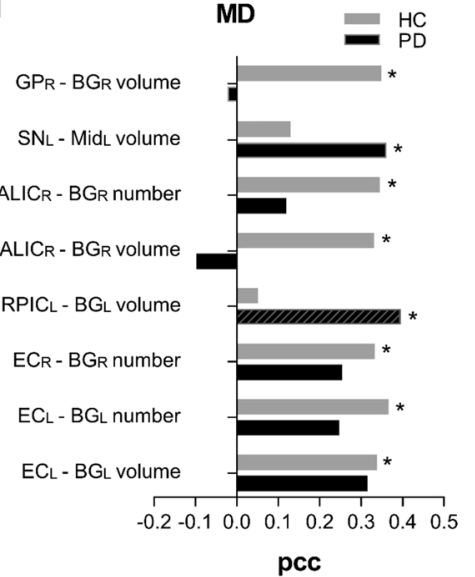

Fig. 4 Fractional anisotropy (FA) values and mean diffusivity (MD) values analysis. $\mathbf{a}$ and $\mathbf{b}$ Comparison of FA and MD values between PD and HC groups. $\mathbf{c}$ and $\mathbf{d}$ Correlations between PVS burden indexes and FA, MD values in PD and HC. * Indicates $p<0.05$, $* *$ indicates $p<0.01$, *** indicates $p<0.001$. The results are presented as means \pm standard error of mean. Abbreviations: vCa ventral caudate, dCa dorsal caudate, vmPu ventromedial putamen, dIPu dorsolateral putamen, GP globus pallidus, SN substantia nigra, ALIC anterior limb of internal capsule, PLIC posterior limb of internal capsule, RPIC retrolenticular part of internal capsule, EC external capsule, CP cerebral peduncle, CT corticospinal tract, pcc partial correlation coefficient.

in the PD patients. We suggested that the PVS number in left BG could reflect severity and progression of PD motor symptoms. In addition, our results showed that PD patients with more severe PVS burden in right hemisphere needed higher dosage of anti-PD drugs, which is in line with the hypothesis that possible dysfunction of the PVS pathway existed in PD, as well as the incomplete response to levodopa caused by PVS-related dysfunction of presynaptic nigrostriatal dopaminergic system ${ }^{25,26}$. The right striatum might have lower quantities of dopamine than those of left, which lead to vulnerability to nigrostriatal denervation in right-hemisphere ${ }^{38}$. Interestingly, PD patients with relatively heavier right-hemisphere dysfunction displayed greater medication doses ${ }^{39}$, which was consistent with our results. Besides, since only the PVS number of BG was correlated to these clinical features, the PVS number rather than PVS volume in BG might be a more important measure to represent severity of motor symptoms, which was consistent with one previous study declaring that PVS number was more meaningful than PVS volume ${ }^{21}$.

A previous study reviewed that the prefrontal-limbic network in depression is modulated by hypothalamus, BG, and midbrain ${ }^{40}$. Increased PVS burden over time significantly associated with higher incidence of depressive symptoms ${ }^{6}$ and might be able to predict resistance to antidepressant monotherapy in elderly patients with depression ${ }^{41}$. An animal study supported this assumption with revealing that dilated PVS might exacerbate depression-like behaviors by increasing the levels of inflammatory factors ${ }^{42}$. We found significant association between HAMD score and PVS burden of both BG and midbrain in the HC group, indicating that poor emotional states like depression might aggravate the PVS burden. But the emotional status of most HCs was at a normal level, the correlation might be in different extent while focusing on patients with depression. As PVS dysfunction might relate to neuro-neurophysiology and clinical motor features of PD, it also related to cognitive decline in $P D^{3,4,15}$. The PVS burden, particularly correlated with executive functioning $^{4}$ and visuospatial ability ${ }^{43}$, which were frequently involved cognitive domains in PD. Possible mechanism of PVS might be decreased clearance of metabolic waste, lead to accumulation of $A \beta$, a-synuclein relating to cognitive decline. In patients with cognition decline, brain atrophy was often accompanied by cerebrospinal fluid (CSF) space enlargement and cerebral ventricular dilatation, might following by a secondary ex-vacuo effect as compensation ${ }^{3,44}$. Therefore, the PVS might be an earlier imaging feature than brain atrophy during the aging and cognition decline process ${ }^{3}$. Studies of PD provided evidence that PVS burden in BG could be regarded as biomarker to evaluate cognitive decline in PD ${ }^{15,30}$. We also found that healthy elderly with more severe PVS burden in midbrain, had more decreased cognitive function. However, the PVS burden did not show a significant correlation with the emotional state and cognitive function in PD patients, which might be caused by the possible presence of other influential factors that covered these correlations.

PVS is a microscopic tubular structure that occupies extravascular space, in which water molecules move freely ${ }^{45}$. Regarding to white matter fiber tracts, they are spatially parallelizing with PVSs ${ }^{46}$. Except for vessels, water molecules inside the PVS are also hindered by surrounding brain tissue. DTI-derived features reflect the diffusion properties of both the surrounding brain tissue and fluid from PVS, thus the enlargement of PVS might have substantial influence on DTI features ${ }^{45}$. At the same time, the activity of the glymphatic system could be evaluated by calculating diffusivity along the PVS, providing more evidence that the PVS burden related to the impairment of white matter fiber ${ }^{47}$. In addition to the relationship between PVS and 
a
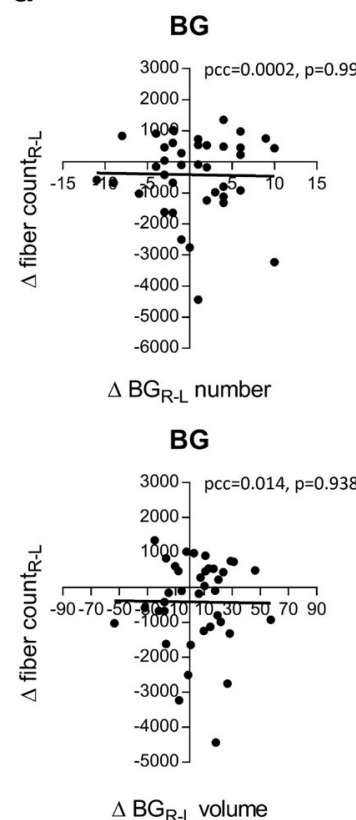

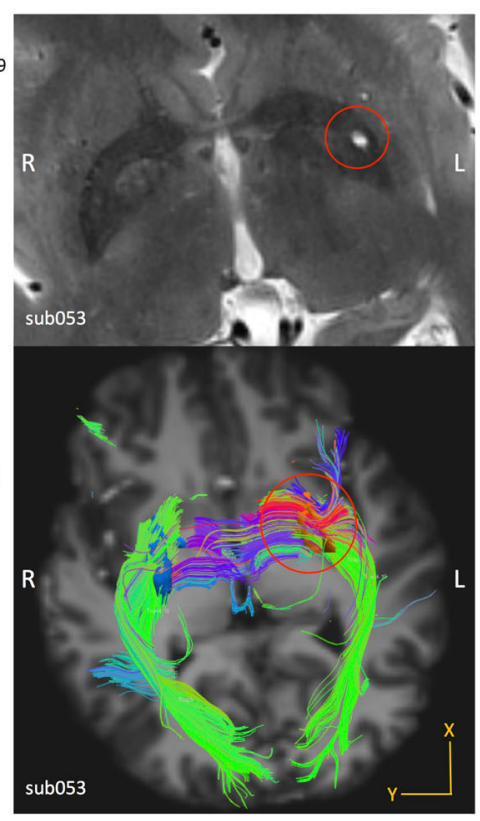

b

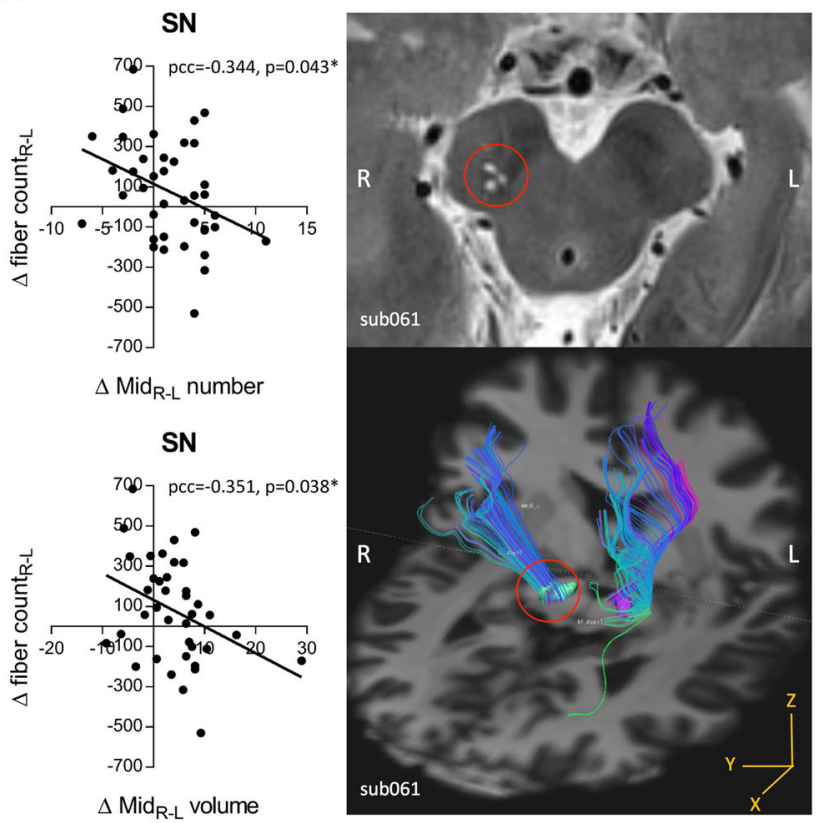

Fig. 5 White matter fibers tacking analysis. a Correlations of the difference between bilateral PVS burden indexes in $B G\left(\Delta B G_{R-L}\right.$ number and volume) and the difference between fiber counts of white fiber tract passing through the bilateral BG in PD. An example of fiber tacking result of one PD patient (sub053) with severer PVS burden in left-hemispheric putamen. b Correlations of the difference between bilateral PVS burden indexes in midbrain ( $\Delta \mathrm{Mid}_{\mathrm{R}-\mathrm{L}}$ number and volume) and the difference between fiber counts of white fiber tract passing through the bilateral midbrain in PD. An example of fiber tacking result of one PD patient (sub061) showing severe PVS burden and sparser fiber in right midbrain. * Indicates the partial correlation coefficient (pcc) with $p<0.05$. $\Delta$ fiber count $\mathrm{R}_{\mathrm{R}-\mathrm{L}}$ difference between fiber counts of white fiber tract passing through the bilateral BG or midbrain.

dopaminergic terminals that we have mentioned above ${ }^{11}$, gray and white matter microstructures might also be an important part of the pathophysiological mechanism of PVS.

We used the FA value to quantify the degree of directional dependence of diffusion that depends on the number and density of axons ${ }^{48}$. The MD value was also used to describe the overall amount of isotropic diffusion within brain tissue ${ }^{49}$, and its increasing might be caused by enlargement of extracellular space, suggesting degeneration of brain tissue $e^{50}$. However, towards the changes of white matter microstructure in PD, the alterations of FA and MD due to PD remain controversial. Most studies focusing on advanced-stage PD patients reported decreased FA and/or increased $M D$, indicating demyelination and axonal degeneration of white matter microstructure ${ }^{51}$. Other results like increased FA and decreased MD were defined as microstructural compensation in prodromal-stage and early-stage of PD, which was distinguished from pathological findings ${ }^{52}$. Our results showed significantly decreased FA in caudate accompanied by increased $M D$ in caudate and putamen, demonstrating degeneration in these respective regions. And significantly increased FA and MD values in GP and ALIC were detected, indicating possible selective neurodegeneration or a potential compensatory reorganization after treatment ${ }^{50}$.

Back to the correlation between PVS burden and surrounding brain tissues, the PVS number in BG was correlated with the load of deep white matter hyperintensity, which was corresponding to the demyelination and sparseness of axons ${ }^{21}$. And some PD cases reported that the DTI features of fibers adjacent to enlarged PVS were changed compared to the normal contralateral fibers ${ }^{28}$. Similarly, our results indicated that PVS burden severity was related to the integrity of adjacent white matter fibers. The participants with higher PVS volume in midbrain had a more decreased FA and more increased MD in SN, revealed that the PVS might increase the diffusivity of water molecules and even exacerbate the degeneration of SN. The MD values of GP, ALIC,
RPIC, and EC were also increasing with the PVS burden indexes of $B G$, indicating PVS burden in BG also influenced the adjacent white matter microstructure. We also investigated the correlation between the fiber counts of white matter fiber track passing through bilateral subcortical nuclei and bilateral PVS burden. For the midbrain in PD patients, we found that the hemisphere with more severe PVS burden in midbrain showed a sparser fiber track passing through SN than the contralateral hemisphere. Since the HCs had less severe PVS burden than PD patients, they just showed a negative correlation trend, with no statistical significance was attained. PVS burden in unilateral cerebral hemisphere was significantly correlated to ipsilateral DTI-derived features, while the same index in contralateral hemisphere only showed a correlation trend. Possibly, we quantitated the PVS burden in the whole BG and midbrain, within which the distribution of PVS might vary in different subcortical nuclei and white matter fibers. Although without statistical significance, these results still indicating slightly positive or negative correlations between PVS burden and both gray and white matter microstructure. Based on these results, smaller PVS burden that was previously considered as type 1 "normal" structure might still exhibit physiological dysfunctions, which was mainly reflected by causing an indispensable important effect on the brain parenchyma, particularly the DTI-derived features. However, this phenomenon might not be unique to PD. As a possible biomarker for cerebral small-vessel disease affecting nearby brain parenchyma ${ }^{21}$, PVS burden indexes might be able to play a disease-modifying role in a certain disease by involving related brain regions.

Growing evidence had shown that insufficient clearance of pathologic protein deposits through brain drainage system including the PVS pathway might serve as new targets for promising modifier treatment and even prevention for neurodegenerative disorders ${ }^{53}$. The "perivascular pump" driven by arterial pulsation might be a powerful convection enhanced delivery method, by which viral particles for gene-therapy or therapeutic 
agents could be administered directly into central nervous system ${ }^{54}$. Furthermore, focused ultrasound combined with microbubbles could enhance the brain blood barrier opening along with expansion and contraction of PVS, which might induce bulk flow and lead to increased penetration of drugs into the brain tissue $\mathrm{e}^{55}$. Another important therapeutic target is enhancing the brain clearance mechanisms by reducing in reactive astro- and microgliosis, normalizing the perivascular aquaporin-4 polarization, inducing fibrinolysis of fibrin clots in PVS, which are still beyond clinical application so $\mathrm{far}^{56}$. Since the clearance of interstitial solutes increases by $60 \%$ during the sleep state and exercise could also increase glymphatic influx and clearance, sleep improvement, and voluntary exercise provide easily implemented strategies to improve glymphatic function ${ }^{56}$. Moreover, increased PVS burden is associated with hypertension ${ }^{56}$, thus, improved hypertension control might also help in slowing down the aggravation of PVS burden and its potential role in neurodegenerative disorders.

In conclusion, the present study demonstrated that the PVS burden in the BG and the midbrain were already aggravated in early-stage PD patients. The PVS burden indexes, including PVS number and volume, could be considered as a biomarker to evaluate severity and progression of PD motor symptom, and aid in predicting medication dosage. Furthermore, the PVS burden might also reflect microstructural alteration of surrounding brain tissues. Despite the limited sample size, with higher spatial resolution and SNR of MRI data, our study provides preliminary evidence to support the potential role of PVS in the pathophysiology of PD. Future work will focus on reviewing the correlations between PVS burden and the progression of clinical feature, as well as the continuing impact of PVS burden on brain parenchyma through longitudinal research during the course of PD.

\section{METHODS}

\section{Participants and clinical data collection}

This study was approved by the ethics committee of Second Affiliated Hospital of Zhejiang University School of Medicine. Written informed consent was obtained from all the participants. We recruited 40 PD patients from the outpatient clinic of the Department of Neurology of Second Affiliated Hospital of Zhejiang University School of Medicine, and 41 age-matched and sex-matched healthy controls (HCs) from neighboring communities.

PD patients met the Movement Disorder Society (MDS) Clinical Diagnostic Criteria for $\mathrm{PD}^{57}$. HCs had no history of neurological or psychiatric disease and no family history of PD or related neurodegenerative disorders. Participants with small vessel disease such as white matter hyperintensity, lacunar infarctions, and cerebral microbleeds were excluded. All the participants underwent a clinical evaluation, including clinical histories (self-report or medical records), neurological, and neuropsychological examinations. PD disease severity was assessed using MDS-UPDRS and Hoehn \& Yahr stage ( $\mathrm{H}-\mathrm{Y}$ stage) during off-stage, earlystage PD was defined as $\mathrm{H}-\mathrm{Y}$ stage between 1 and $2.5^{58}$. We separately analyzed the MDS-UPDRS subscores for more detailed information, including the subscore I for non-motor experiences of daily living, subscore II for motor experiences of daily living, subscore III for motor examination, and subscore IV for motor complications ${ }^{59}$. We applied the HAMA and HAMD to assess emotional status, the MMSE, MoCA, and PANDA to evaluate cognitive function.

\section{MRI images acquisition}

MRI images were acquired with a $7 \mathrm{~T}$ Magnatom research system (Siemens Healthcare, Erlangen, Germany) with prototype sequences, including a magnetization prepared with two rapid gradient echoes (MP2RAGE) sequence (voxel size: $0.7 \times 0.7 \times 0.7 \mathrm{~mm}^{3}, \mathrm{TR}=5000 \mathrm{~ms}, \mathrm{TI} 1 / \mathrm{TL} 2=900$ / $2750 \mathrm{~ms}, \mathrm{TE}=2.3 \mathrm{~ms}, a 1 / a 2=5^{\circ} / 3^{\circ}$, and two times generalized autocalibrating partial parallel acquisition (GRAPPA) acceleration), a T2-weighted turbo spin echo (TSE) sequence (voxel size: $0.5 \times 0.5 \times 2.4 \mathrm{~mm}^{3}, \mathrm{TR}=$ $7000 \mathrm{~ms}$, and $\mathrm{TE}=66 \mathrm{~ms}$ ) and a multiband echo-planar DTI sequence (voxel size: $1.5 \times 1.5 \times 1.5 \mathrm{~mm}, \mathrm{TR}=6000 \mathrm{~ms}, \mathrm{TE}=87.4 \mathrm{~ms}$ ). A patient underwent an extra 3T MRI scan to obtain another T2 image on a 3T Prisma research system (Siemens Healthcare, Erlangen, Germany) using a T2-weighted TSE sequence (voxel size: $0.5 \times 0.5 \times 2.8 \mathrm{~mm}^{3}, \mathrm{TR}=9360 \mathrm{~ms}$, and $\mathrm{TE}=100 \mathrm{~ms}$ ) for comparsion.

\section{MRI images processing}

In this study, we focused on the effects of small size PVSs. Therefore, a PVS was defined as a small $(<3 \mathrm{~mm})$, smooth, linear-shaped, ovoid-shaped, or round-shaped space with isointense CSF that ran along with perforating arteries $^{60-62}$. We acquired a high-resolution MP2RAGE image to visualize the PVS system and its spatially corresponding lenticulostriate artery (LAS) simultaneously, as shown in Fig. 1c, with PVSs showing low intensity similar to CSF and the blood vessels showing high intensity. The PVS burden was defined to represent the severity of PVSs, which was assessed on axial T2-weighted images in the BG and midbrain (Fig. 1b). According to previous studies ${ }^{15,63}$, we performed PVS quantification in the slice containing the maximum amount of PVSs in the BG region ${ }^{60}$, of which the anterior and posterior border zones were the anterior end of the insula and the posterior end of the thalamus ${ }^{1}$. For the midbrain region, the PVSs were counted in the whole volume ${ }^{64}$. The PVS counting, segmentation, and volume calculation were performed using the ITK-SNAP software version 3.8 (http://www.itksnap.org/). We separately counted PVS number and calculated the PVS volume in four defined regions: $B G_{R}, B G_{L}, M_{R}$, and Mid $_{\llcorner}$. We manually delineated along the boundary of all identified PVSs. The ITK-SNAP software automatically gave the voxel number of identified PVSs in each region, and the PVS volume was calculated as the sum of the individual volume of identified PVS in each region per $\mathrm{mm}^{3}$. Then we obtained the PVS number and the PVS volume of each region, regarded as the PVS burden indexes.

Total brain volume that was expressed as the sum of total gray and white matter volume was estimated on MP2RAGE images using VBM8 embedded in SPM8 software (Statistical Parametric Mapping; https://www. fil.ion.ucl.ac.uk/spm/software/spm8/). The DTI data was processed using FSL version 6.0.1 (FMRIB Software Library; http://www.fmrib.ox.ac.uk/fsl) to generate fractional anisotropy (FA) and mean diffusivity (MD) maps. The Brainnetome Atlas ${ }^{65}$ adding additional nuclei within midbrain, and the ICBM-DTI-81 white matter atlas ${ }^{66}$ were separately transformed into each participant's DTI space ${ }^{67}$. Then we used "fsImeants" command of FSL to obtain 12 subcortical nuclei ROls, including the bilateral $\mathrm{vCa}, \mathrm{dCa}, \mathrm{vmPu}$, dIPu, GP and SN, as well as 12 FOls, including the bilateral ALIC, posterior limb of internal capsule (PLIC), RPIC, EC, CP, and corticospinal tract (CT) from these two atlases. Then the averages of $F A$ and $M D$ values were extracted for subsequent analysis.

Deterministic tractography was conducted using Diffusion Toolkit version 0.6.4 and TrackVis version 0.6.1 (http://trackvis.org) in each participant's DTI space. We applied a DWI mask threshold and an angular threshold of $35^{\circ}$ to limit deviations from main path. We loaded ROIs from transformed atlas including bilateral vCa, dCa, vmPu, dIPu, GP, and SN to track white matter fibers passing through these subcortical nuclei, and the fiber counts of each track were given for subsequent analysis.

\section{Statistical analysis}

The results were expressed as mean \pm standard error of mean (SEM) for continuous variables and as percentages for the categorical variables. The Student's two-sample $t$-test was used for the comparison of continuous variables including clinical and DTI features between groups. The chisquared test was used for the comparison of categorical variables. The difference between right- and left-hemispheric PVS burden indexes, and the differences between fiber counts of white matter fibers passing through the right- and left-hemispheric nuclei within BG and midbrain were also calculated. Partial correlation analysis was performed to calculate pcc and evaluate the correlations between PVS number, PVS volume and MDS-UPDRS, H-Y stage, LEDD, MMSE, MoCA, PANDA, HAMA, HAMD scores, and DTI parameters. Age, sex, education, and total brain volume were entered as covariates due to their potential influence. These analyses were considered significant at $p<0.05$, uncorrected. At the same time, we further used a FDR corrected $p<0.05$ for multiple testing.

Statistical analyses were conducted with SPSS Statistics software version 22 (IBM Corporation, Armonk, NY, USA). Statistical plots were generated by GraphPad Prism version 7 (GraphPad Inc., San Diego, CA, USA). 


\section{Reporting summary}

Further information on research design is available in the Nature Research Reporting Summary linked to this article.

\section{DATA AVAILABILITY}

Clinical and neuroimaging data can be shared on reasonable request from qualified investigators by contacting the corresponding authors. Sharing and reuse of our data require the expressed written permission of the authors.

\section{CODE AVAILABILITY}

As previously mentioned in the "MRI images processing" subsection, the PVS quantification was performed using the ITK-SNAP version 3.8 (http://www.itksnap. org/). Total brain volume was estimated using VBM8 embedded in SPM8 software (Statistical Parametric Mapping; https://www.fil.ion.ucl.ac.uk/spm/software/spm8/). The DTI data was processed using FSL 6.0.1 (FMRIB Software Library; http://www. fmrib.ox.ac.uk/fsl). Deterministic tractography was conducted using Diffusion Toolkit 0.6.4 and TrackVis 0.6.1 (http://trackvis.org). Statistical analyses were conducted with SPSS Statistics software version 22 (IBM Corporation, Armonk, NY, USA). Statistical plots were generated by GraphPad Prism version 7 (GraphPad Inc., San Diego, (A, USA).

Received: 12 August 2020; Accepted: 22 December 2020; Published online: 05 February 2021

\section{REFERENCES}

1. Bouvy, W. H. et al. Perivascular spaces on 7 Tesla brain MRI are related to markers of small vessel disease but not to age or cardiovascular risk factors. J. Cereb. Blood Flow Metab. 36, 1708-1717 (2016).

2. Adams, H. H. et al. A priori collaboration in population imaging: The Uniform Neuro-Imaging of Virchow-Robin Spaces Enlargement consortium. Alzheimer's Dement. 1, 513-520 (2015).

3. Niazi, M. et al. Quantitative MRI of perivascular spaces at 3T for early diagnosis of mild cognitive impairment. Am. J. Neuroradiol. 39, 1622-1628 (2018).

4. Passiak, B. S. et al. Perivascular spaces contribute to cognition beyond other small vessel disease markers. Neurology 92, e1309-e1321 (2019).

5. Berezuk, C. et al. Virchow-Robin spaces: correlations with polysomnographyderived sleep parameters. Sleep 38, 853-858 (2015).

6. van Sloten, T. T. et al. Cerebral small vessel disease and association with higher incidence of depressive symptoms in a general elderly population: The AGESReykjavik Study. Am. J. Psychiatry 172, 570-578 (2015).

7. Potter, G. M. et al. Enlarged perivascular spaces and cerebral small vessel disease. Int. J. Stroke 10, 376-381 (2015).

8. Banerjee, G. et al. MRI-visible perivascular space location is associated with Alzheimer's disease independently of amyloid burden. Brain 140, 1107-1116 (2017).

9. Favaretto, A. et al. Enlarged Virchow Robin spaces associate with cognitive decline in multiple sclerosis. PLOS ONE 12, ARTN https://doi.org/ e018562610.1371/journal.pone.0185626 (2017).

10. Laitinen, L. V. et al. Dilated perivascular spaces in the putamen and pallidum in patients with Parkinson's disease scheduled for pallidotomy: a comparison between MRI findings and clinical symptoms and signs. Mov. Disord. 15, 1139-1144 (2000).

11. Lee, D., Hong, I. K. \& Ahn, T. B. Dilated Virchow-Robin space and dopamine transporter imaging in the striatum of patients with Parkinsonism. Can. J. Neurol. Sci. Le. J. Can. Sci. Neurol. 42, 248-254 (2015).

12. Shen, T. et al. Early-onset Parkinson's disease caused by PLA2G6 compound heterozygous mutation, a case report and literature review. Front. Neurol. 10, 915 (2019).

13. Kalia, L. V. \& Lang, A. E. Parkinson's disease. Lancet 386, 896-912 (2015).

14. Fenelon, G. et al. Parkinsonism and dilatation of the perivascular spaces (etat crible) of the striatum: a clinical, magnetic resonance imaging, and pathological study. Mov. Disord. 10, 754-760 (1995).

15. Park, Y. W. et al. Magnetic resonance imaging-visible perivascular spaces in basal ganglia predict cognitive decline in Parkinson's disease. Mov. Disord. https://doi. org/10.1002/mds.27798 (2019).

16. Weller, R. O., Djuanda, E., Yow, H. Y. \& Carare, R. O. Lymphatic drainage of the brain and the pathophysiology of neurological disease. Acta Neuropathol. 117, 1-14 (2009).

17. Wuerfel, J. et al. Perivascular spaces-MRI marker of inflammatory activity in the brain? Brain 131, 2332-2340 (2008).
18. Iliff, J. J. et al. A paravascular pathway facilitates CSF flow through the brain parenchyma and the clearance of interstitial solutes, including amyloid beta. Sci. Transl. Med. 4, 147ra111 (2012).

19. Sundaram, S. et al. Establishing a framework for neuropathological correlates and glymphatic system functioning in Parkinson's disease. Neurosci. Biobehav. Rev. 103, 305-315 (2019).

20. Gonzalez-Castro, V. et al. Reliability of an automatic classifier for brain enlarged perivascular spaces burden and comparison with human performance. Clin. Sci. 131, 1465-1481 (2017).

21. Laveskog, A. et al. Perivascular spaces in old age: assessment, distribution, and correlation with white matter hyperintensities. Am. J. Neuroradiol. 39, 70-76 (2018).

22. Cherian, I. et al. Exploring the Virchow-Robin spaces function: a unified theory of brain diseases. Surg. Neurol. Int. 7, S711-S714 (2016).

23. Ramirez, J. et al. Imaging the perivascular space as a potential biomarker of neurovascular and neurodegenerative diseases. Cell. Mol. Neurobiol. 36, 289-299 (2016).

24. Li, Y. et al. Dilated perivascular space in the midbrain may reflect dopamine neuronal degeneration in Parkinson's disease. Front. Aging Neurosci. 12, 161 (2020).

25. Mehta, S. H. et al. Dilated Virchow-Robin spaces and Parkinsonism. Mov. Disord. 28, 589-590 (2013).

26. Mestre, T. A. et al. Can isolated enlarged Virchow-Robin spaces influence the clinical manifestations of Parkinson's disease? Mov. Disord. Clin. Pract. 1, 67-69 (2014).

27. Mancardi, G. L. et al. Lacunae and cribriform cavities of the brain. Correlations with pseudobulbar palsyand Parkinsonism. Eur. Neurol. 28, 11-17 (1988).

28. Conforti, R. et al. Dilated Virchow-Robin space and Parkinson's disease: a case report of combined MRI and diffusion tensor imaging. Radiol. Case Rep. 13, 871-877 (2018).

29. Kim, D. G., Oh, S. H. \& Kim, O. J. A case of disseminated polycystic dilated perivascular spaces presenting with dementia and parkinsonism. J. Clin. Neurol. 3, 96-100 (2007).

30. Shibata, K., Sugiura, M., Nishimura, Y. \& Sakura, H. The effect of small vessel disease on motor and cognitive function in Parkinson's disease. Clin. Neurol. Neurosurg. 182, 58-62 (2019).

31. Wan, Y. et al. Exploring the association between Cerebral small-vessel diseases and motor symptoms in Parkinson's disease. Brain Behav. 9 https://doi.org/ 10.1002/brb3.1219 (2019).

32. Serrien, D. J., Ivry, R. B. \& Swinnen, S. P. Dynamics of hemispheric specialization and integration in the context of motor control. Nat. Rev. Neurosci. 7, 160-166 (2006).

33. Lizarraga, K. J. et al. Asymmetric neuromodulation of motor circuits in Parkinson's disease: the role of subthalamic deep brain stimulation. Surg. Neurol. Int. 8, 261 (2017).

34. Scherfler, C. et al. Left hemispheric predominance of nigrostriatal dysfunction in Parkinson's disease. Brain 135, 3348-3354 (2012).

35. Iranzo, A. et al. Left-hemispheric predominance of nigrostriatal deficit in isolated REM sleep behavior disorder. Neurology 94, e1605-e1613 (2020).

36. Fling, B. W. et al. Asymmetric pedunculopontine network connectivity in parkinsonian patients with freezing of gait. Brain 136, 2405-2418 (2013).

37. Lam, S. et al. Does dominant pedunculopontine nucleus exist? Brain 138, e323 (2015).

38. Haaxma, C. A. et al. Side of symptom onset affects motor dysfunction in Parkinson's disease. Neuroscience 170, 1282-1285 (2010).

39. Hanna-Pladdy, B., Pahwa, R. \& Lyons, K. E. Paradoxical effect of dopamine medication on cognition in Parkinson's disease: relationship to side of motor onset. J. Int. Neuropsychol. Soc. 21, 259-270 (2015).

40. Bennett, M. R. The prefrontal-limbic network in depression: modulation by hypothalamus, basal ganglia and midbrain. Prog. Neurobiol. 93, 468-487 (2011).

41. Patankar, T. F. et al. Virchow-Robin space dilatation may predict resistance to antidepressant monotherapy in elderly patients with depression. J. Affect. Disord. 97, 265-270 (2007)

42. Li, Y. et al. Dilated Virchow-Robin spaces in the hippocampus impact behaviors and effects of anti-depressant treatment in model of depressed rats. J. Affect. Disord. 219, 17-24 (2017).

43. Maclullich, A. M. et al. Enlarged perivascular spaces are associated with cognitive function in healthy elderly men. J. Neurol. Neurosurg. Psychiatry 75, 1519-1523 (2004).

44. Rudie, J. D., Rauschecker, A. M., Nabavizadeh, S. A. \& Mohan, S. Neuroimaging of dilated perivascular spaces: from benign and pathologic causes to mimics. $J$. Neuroimaging 28, 139-149 (2018).

45. Sepehrband, F. et al. Perivascular space fluid contributes to diffusion tensor imaging changes in white matter. Neuroimage 197, 243-254 (2019).

46. Cai, K. et al. The feasibility of quantitative MRI of perivascular spaces at 7T. J. Neurosci. Methods 256, 151-156 (2015). 
47. Taoka, T. et al. Evaluation of glymphatic system activity with the diffusion MR technique: diffusion tensor image analysis along the perivascular space (DTIALPS) in Alzheimer's disease cases. Jpn. J. Radiol. 35, 172-178 (2017).

48. Zasler, N. D. \& Kaplan, P. E. Fractional Anisotropy (Springer: New York, 2016)

49. Vos, S. B. et al. The influence of complex white matter architecture on the mean diffusivity in diffusion tensor MRI of the human brain. Neurolmage 59, 2208-2216 (2012).

50. Atkinson-Clement, C., Pinto, S., Eusebio, A. \& Coulon, O. Diffusion tensor imaging in Parkinson's disease: review and meta-analysis. Neurolmage 16, 98-110 (2017).

51. Andica, C. et al. Neurocognitive and psychiatric disorders-related axonal degeneration in Parkinson's disease. J. Neurosci. Res. 98, 936-949 (2020).

52. Sanjari Moghaddam, H., Dolatshahi, M., Mohebi, F. \& Aarabi, M. H. Structural white matter alterations as compensatory mechanisms in Parkinson's disease: a systematic review of diffusion tensor imaging studies. J. Neurosci. Res. https://doi. org/10.1002/jnr.24617 (2020).

53. Pahnke, J., Langer, O. \& Krohn, M. Alzheimer's and ABC transporters-new opportunities for diagnostics and treatment. Neurobiol. Dis. 72 Part A, 54-60 (2014).

54. Hadaczek, P. et al. The "perivascular pump" driven by arterial pulsation is a powerful mechanism for the distribution of therapeutic molecules within the brain. Mol. Ther. 14, 69-78 (2006).

55. Chen, $H$. et al. Focused ultrasound-enhanced intranasal brain delivery of brainderived neurotrophic factor. Sci. Rep. 6 https://doi.org/10.1038/Srep28599 (2016).

56. Mestre, H., Kostrikov, S., Mehta, R. I. \& Nedergaard, M. Perivascular spaces, glymphatic dysfunction, and small vessel disease. Clin. Sci. 131, 2257-2274 (2017).

57. Postuma, R. B. et al. MDS clinical diagnostic criteria for Parkinson's disease. Mov. Disord. 30, 1591-1599 (2015).

58. Kwon, D. Y., Kwon, Y. \& Kim, J. W. Quantitative analysis of finger and forearm movements in patients with off state early stage Parkinson's disease and scans without evidence of dopaminergic deficit (SWEDD). Parkinsonism Relat. Disord. 57, 33-38 (2018).

59. Goetz, C. G. et al. Movement Disorder Society-sponsored revision of the Unified Parkinson's Disease Rating Scale (MDS-UPDRS): scale presentation and clinimetric testing results. Mov. Disord. 23, 2129-2170 (2008).

60. Liang, Y. et al. Enlarged perivascular spaces in the centrum semiovale are associated with poststroke depression: a 3-month prospective study. J. Affect. Disord. 228, 166-172 (2018).

61. Wardlaw, J. M. et al. Neuroimaging standards for research into small vessel disease and its contribution to ageing and neurodegeneration. Lancet Neurol. 12, 822-838 (2013).

62. Jimenez-Balado, J. et al. Prevalence of hippocampal enlarged perivascular spaces in a sample of patients with hypertension and their relation with vascular risk factors and cognitive function. J. Neurol. Neurosurg. Psychiatry 89, 651-656 (2018).

63. Zhang, C. Q. et al. Risk factors of dilated Virchow-Robin spaces are different in various brain regions. PLOS ONE 9 https://doi.org/10.1371/journal.pone.0105505 (2014).

64. Dubost, F. et al. Enlarged perivascular spaces in brain MRI: automated quantification in four regions. Neuroimage 185, 534-544 (2019).

65. Fan, L. et al. The human Brainnetome Atlas: a new Brain Atlas based on connectional architecture. Cereb. Cortex 26, 3508-3526 (2016).

66. Mori, S. et al. Stereotaxic white matter atlas based on diffusion tensor imaging in an ICBM template. Neurolmage 40, 570-582 (2008).

67. Cui, Z. et al. PANDA: a pipeline toolbox for analyzing brain diffusion images. Front. Hum. Neurosci. 7, 42 (2013).

\section{ACKNOWLEDGEMENTS}

This work was supported by grants from the National Key R\&D Program of China (2018YFA0701400), the National Natural Science Foundation of China (61673346, 81520108010), the National Major Scientific Instruments Development Project of China (81527901), the Science and Technology Project of Zhejiang Province (2016C34006), the Fundamental Research Funds for the Central Universities (2019XZZX001-01-21) and Zhejiang Lab (No. 2018EB0ZX01). The authors gratefully thank all study participants for their participation in this study. And we would like to acknowledge Zhejiang University 7 T Brain Imaging Research Center for all the help and support.

\section{AUTHOR CONTRIBUTIONS}

T.S., H.-Y.L., and B.R.Z. designed this study. Y.X.C., J.T., and W.L. were responsible for diagnosis and clinical evaluation. Y.-C.H. and T.K. designed MRI sequences. C.-Y.L. contributed to the DTI sequence modification. Y.M.Y., J.J.X., and S.Z. collected MRI data. T.S. and Y.M.Y. analyzed MRI data. T.S. wrote the manuscript. H.-Y.L. and B.R.Z. contributed to the revision of the manuscript.

\section{COMPETING INTERESTS}

The authors declare no competing interests.

\section{ADDITIONAL INFORMATION}

Supplementary information The online version contains supplementary material available at https://doi.org/10.1038/s41531-021-00155-0.

Correspondence and requests for materials should be addressed to B.Z. or H.-Y.L.

Reprints and permission information is available at http://www.nature.com/ reprints

Publisher's note Springer Nature remains neutral with regard to jurisdictional claims in published maps and institutional affiliations.

(i) Open Access This article is licensed under a Creative Commons Attribution 4.0 International License, which permits use, sharing, adaptation, distribution and reproduction in any medium or format, as long as you give appropriate credit to the original author(s) and the source, provide a link to the Creative Commons license, and indicate if changes were made. The images or other third party material in this article are included in the article's Creative Commons license, unless indicated otherwise in a credit line to the material. If material is not included in the article's Creative Commons license and your intended use is not permitted by statutory regulation or exceeds the permitted use, you will need to obtain permission directly from the copyright holder. To view a copy of this license, visit http://creativecommons. org/licenses/by/4.0/.

(c) The Author(s) 2021 